\title{
The Proportionate APL-I algorithm
}

\author{
Felix Albu \\ Department of Electronics, Valahia University of Targoviste, Targoviste, Romania \\ felix.albu@valahia.ro
}

\begin{abstract}
A proportionate affine projection like (PAPL-I) adaptive filtering algorithm is proposed that incorporates the proportionate feature to affine projection like (APL-I) algorithm. Simulation results show that the proposed PAPL-I algorithm provides improved steady state performance than the APL-I and affine projection algorithms (APA) in case of an acoustic echo cancellation application with a very sparse echo path. In addition, the PAPL-I algorithm also offers improved performance over the APL-I algorithm for adaptive feedback cancellation for hearing aids systems.
\end{abstract}

Keywords—affine-projection-like principle; adaptive feedback cancellation;

algorithm; proportionate cancellation; acoustic echo

\section{INTRODUCTION}

The conventional least-mean-square (LMS) adaptive-filtering algorithm is very popular because of its simplicity, but its performance is unsatisfactory for colored input signals [1]. The normalized LMS (NLMS) adaptation algorithm offers faster convergence than the conventional LMS algorithm for colored input signals along with computational simplicity [3]. In order to reduce the steady-state misalignment of the NLMS algorithm without compromising its convergence speed, several variable-step-size NLMS algorithms have been proposed (e.g. [3-5]). Although a significant improvement in terms of steady-state misalignment of the NLMS algorithm has been reported the convergence speed of these algorithms for highly correlated input signals continues to be unsatisfactory. In applications that entail highly correlated input signals, the affine-projection algorithm (APA) described by Ozeki and Umeda in [6] offers better convergence speed than NLMS algorithms at the cost of a moderate increase in computational complexity. Following the introduction of the APA in [6], several improved APAs have been proposed (e.g. [3], [5], and [7]). A data-reusing algorithm that does not require the computation of the inverse of the input-signal matrix has been reported in [8]. Other algorithms that do not compute the inverse of the input-signal matrix were reported in [9-13]. A versions in which the input signal is transformed using Gaussian and polynomial kernels have been designed in [14]. Also, an efficient implementation of kernel APA using dichotomous coordinate descent (DCD) iterations [11] was proposed in [15]. Variants with combination of variable projection orders and variable step size were proposed in [16][18].

In [19] a class of affine-projection-like (APL) adaptation algorithms is obtained by eliminating the constraint of forcing to zero the a posteriori error vector in the APA. In this way, direct or indirect inversion of the input signal matrix is not required and, consequently, the amount of computation required per iteration can be reduced [19]. In addition, the proposed APL-I algorithm [19] achieves reduced steady-state misalignment relative to that of APA in system-identification, channel-equalization, and acoustic echo cancelation (AEC) applications.

However, in many practical system identification applications such as echo cancellation, the echo paths are long and sparse. The proportionate normalized least mean square algorithm (PNLMS) [20] and its improved IPNLMS version [21] exploits the sparseness of the echo paths. An improved algorithm, called the $\mu$-law PNLMS, used the logarithm of the coefficient magnitudes instead of coefficient magnitudes [22]. It has been proved that the families of proportionate affine projection algorithms (PAPA) [23-25] have superior performances over the NLMS type of algorithms. Several proportionate based sign algorithms robust to impulsive noise interference have been proposed in [26-28].

In this paper we propose to extend the proportionality principle to the APL-I algorithm. The new type of algorithm is called Proportionate Affine Projection Like (PAPL-I) algorithm. The proportionality coefficients are computed as in [21], but other approaches are possible too (e.g. that of [20] or the logarithmic proportionality of [22]).

The paper is organized as follows. Section II introduces the proposed family of algorithms. The simulation results are presented in Section III for echo cancellation and adaptive feedback cancellation systems. Finally, Section IV concludes this work.

\section{THE PROPOSED PAPL-I ALGORITHM}

The proposed PAPL-I algorithm is derived from the APL-I algorithm proposed in [19]. The adaptive filter that models the true $L$-length echo path, $\mathbf{h}, \quad$ is defined by $\mathbf{w}(k)=\left[w_{0}(k), w_{1}(k), \ldots, w_{L-1}(k)\right]^{T}$, where $k$ is the time index and superscript $T$ denotes transposition. We denote $x(k), z(k)$ and $v(k)$ as the far-end, the near-end and background noise signals, respectively. The desired signal is $y(k)=\mathbf{x}^{T}(k) \mathbf{h}+z(k)+v(k) \quad$ where the vector $\mathbf{x}(k)=[x(k), \ldots, x(k-L+1)]^{T}$, collects the far-end signal. 
The output of the adaptive filter is $\hat{y}(k)=\mathbf{x}^{T}(k) \mathbf{w}(k)$, and the error vector is given as

$$
\mathbf{e}(k)=\mathbf{y}(k)-\mathbf{X}^{T}(k) \mathbf{w}(k),
$$

where $\mathbf{y}(k)=[y(k), y(k-1), \ldots, y(k-M+1)]^{T}$, is a $M \times 1$ vector, $M$ is the projection order, and $\mathbf{X}(k)=[\mathbf{x}(k), \mathbf{x}(k-1), \ldots, \mathbf{x}(k-M+1)]$, is the input signal matrix. The filter coefficients of APL-I algorithm is adapted as follows [19]:

$$
\mathbf{w}(k+1)=\mathbf{w}(k)+\frac{\|\mathbf{X}(k) \mathbf{e}(k)\|^{2}}{\left\|\mathbf{X}^{T}(k) \mathbf{X}(k) \mathbf{e}(k)\right\|^{2}} \mathbf{X}(k) \mathbf{e}(k)
$$

The proportionately principle implies to multiply the update vector with the proportionate matrix $\mathbf{G}(k)=\operatorname{diag}\left\{g_{0}(k), \ldots, g_{L-1}(k)\right\}$, which contains the proportionate factors, $g_{l}(k)$ defined as [19]:

$$
g_{l}(k)=\frac{1-\alpha}{2 L}+\frac{\left|w_{l}(k)\right|(1+\alpha)}{2 \sum_{i=0}^{L-1}\left|w_{i}(k)\right|+\varepsilon}, l=1 . . L,
$$

where $-1<\alpha<1$ and $\varepsilon$ is a small positive constant to avoid division by zero [19]. If we note $\mathbf{P}(k)=\mathbf{G}(k) \mathbf{X}(k)$ we obtain the following weight update of the proportionate APL-I (PAPL-I) algorithm:

$$
\mathbf{w}(k+1)=\mathbf{w}(k)+\frac{\|\mathbf{P}(k) \mathbf{e}(k)\|^{2}}{\left\|\left(\mathbf{X}^{T}(k) \mathbf{P}(k)+\delta \mathbf{I}(k)\right) \mathbf{e}(k)\right\|^{2}} \mathbf{P}(k) \mathbf{e}(k)
$$

The number of multiplications of APL-I is $(3 L+1) M+2 L[19]$, while the number of multiplications of APA is $M^{3}+L M^{2}+(2 L+1) M[32]$. The numerical complexity of PAPL-I in terms of multiplications is given by $(3 L+1) M+4 L$. Therefore the increase in numerical complexity of PAPL-I over APL-I is marginal (i.e. $2 L$ ). It can be easily seen that APA has an increased complexity than PAPL-I and APL-I. For example, for $L=256$ and $M=8$, APA has 21000 multiplications [32], while APL-I has 6664 multiplications [19] and PAPL-I has 7176 multiplications. Therefore, for these particular values, the APL-I and PAPL-I algorithms are about three times less complex than APA.

\section{SIMULATION RESULTS}

The APA, APL-I and PAPL-I algorithms were firstly simulated in an adaptive feedback cancelation (AFC) context. A comprehensive description of these systems can be found in [29]. Suitable algorithms for such systems have been described in [30-31]. In a hearing aid system, the source signal is corrupted by the additive feedback signal generated by the output signal leaking to the input. Also, the hearing-loss has to be considered in order to generate the signals for the adaptive filter and the feedback path was modelled as a 64-tap finite impulse response filter while an exact modelling using an adaptive filter is assumed. The sampling frequency was 16 $\mathrm{kHz}$, a constant gain of $30 \mathrm{~dB}$ in the forward path and a delay of 60 samples were assumed [30]. In the simulations of the APL-I/PAPL-I algorithms $\delta=\varepsilon=10^{-6}$ were used. The step size of APA was chosen in order to match the steady state performance of PAPL-I. For the PAPL-I algorithm $\alpha=0.9$ was used.

In Fig. 1 the input signal was a white noise. It can be seen from Fig. 1 that PAPL-I achieve a smaller steady-state than APL-I. Also, the PAPL-I and APL-I algorithms have a slightly faster convergence rate than APA.

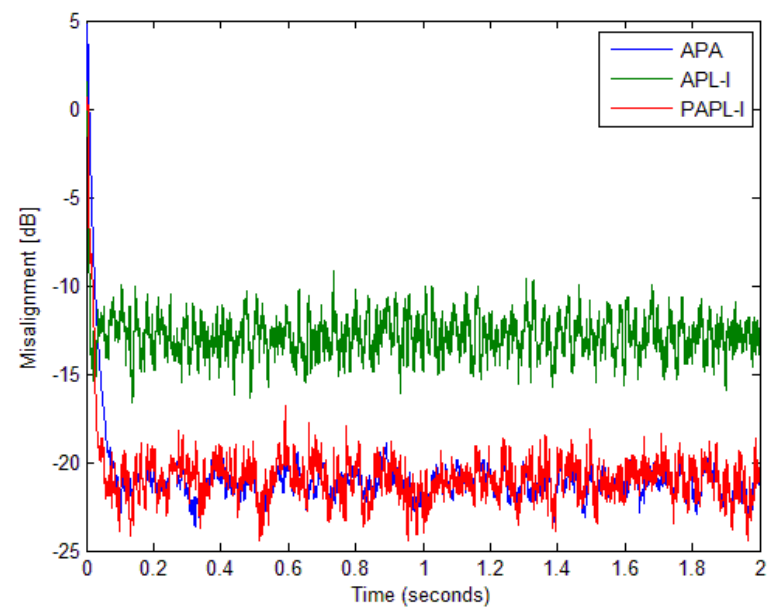

Figure 1. Misalignment of APA, APL-I, and PAPL-I for an AFC application with white noise as input signal, $M=8$.

In Fig. 2 the input signal is a speech signal. It can be seen from Fig. 2 that most of the time, the performance of PAPL-I is superior to that APL-I in terms of misalignment convergence and steady-state performance.

In Fig. 3 the input signal is a music signal. It is well known that this is a difficult case for adaptive feedback cancellation for hearing aids [33]. Figure 3 shows the clear superiority of PAPL-I over APL-I algorithm. Also, it shows that PAPL-I can achieve a faster convergence speed than APA. Figures 1-3 indicates that PAPL-I algorithm can achieve similar or better performances than APA at a fraction of its numerical complexity. 


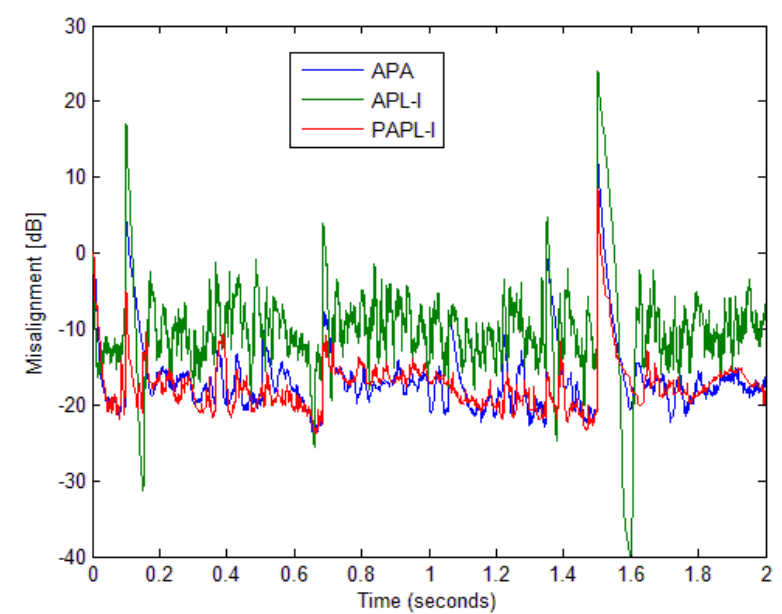

Figure 2. Misalignment of APA, APL-I, and PAPL-I for an AFC application with speech input signal, $M=8$.

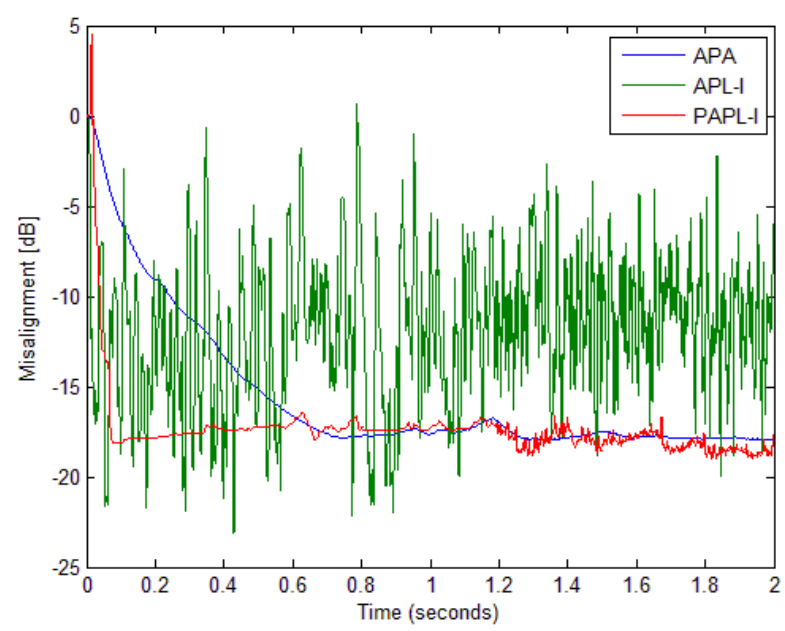

Figure 3. Misalignment of APA, APL-I, and PAPL-I for an AFC application with music input signal, $M=8$.

Next simulations investigate the performance of the APL-I and PAPL-I for an acoustic echo cancellation application. A comparison of APA with APL-I for this application has been presented in [19]. It was shown that the APL-I algorithm offers practically the same steady-state MSD as APA, therefore APA plots are not included in the following pictures. In Fig. 4 the input signal was an auto-regressive AR(1) signal with a pole at 0.95 . A white noise was used as input signal, $M=512$ and $L=$ 3. The performance of the algorithms was measured by the mean square deviation (MSD) in $\mathrm{dB}$ averaged over 10 experiments. In Fig. 4 a very sparse echo path was used (with only 12 non-zero coefficients). Also, the sign of echo path coefficients has been changed after 100000 iterations in order to verify the behavior of the algorithms after a sudden echo path change. It can be noticed that the performance of PAPL-I is better than that of APL-I algorithm. Also, PAPL-I algorithm has better tracking properties than the APL-I algorithm.

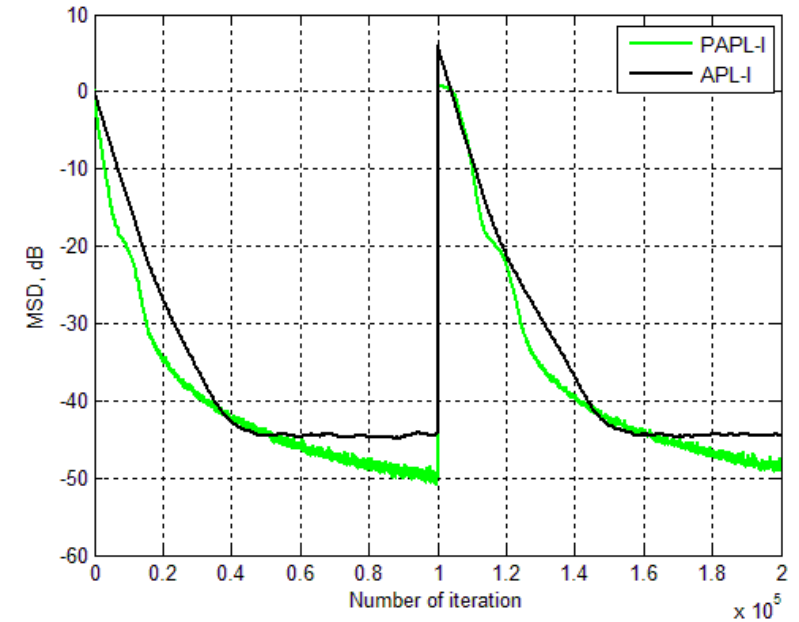

Figure 4: Misalignment of APL-I, and PAPL-I for an AEC application with white noise as input signal using a very sparse echo path and $M=3$.

However, for moderately sparse echo path, the convergence properties of PAPL-I are not as good as those of APL-I, except that of an initial faster convergence speed and better tracking properties (see Fig. 5 after 40000 iterations).

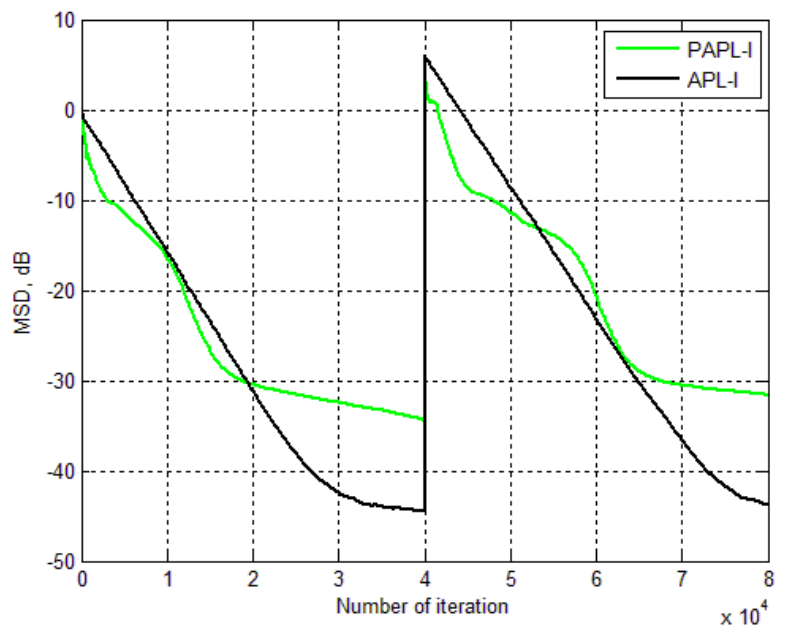

Figure 5: Misalignment of APL-I, and PAPL-I for an AEC application with white noise as input signal using a moderately sparse echo path and $M=3$.

Similar conclusions are obtained in case of dispersive echo paths. These confirm the known behavior of the proportionate versions of numerous types of algorithms whose performance for several applications in case of using dispersive echo paths can be worse than that of the original algorithms (e.g. [19], [22]).

Our future work will be focused investigating the possibility of choosing the right threshold in order to develop a proportionate version of APL-II algorithm proposed in [19]. Also, we'll focus on extending the proportionate idea to a new family of algorithms recently proposed in [34]. 
This work was supported by a grant of the Romanian National Authority for Scientific Research, CNCS-UEFISCDI, project number PN-II-ID-PCE-2011-3-0097.

\section{CONCLUSIONS}

A proportionate APL (PAPL-I) algorithm is developed by incorporating the proportionate feature to the recently proposed APL-I algorithm. Simulations in the context of echo cancellation and adaptive feedback control demonstrated that the PAPL-I algorithm obtains superior performance relative to the APL-I algorithm in several situations, especially in cases where the underlying system is known to be sparse.

\section{REFERENCES}

[1] P.S.R. Diniz, Adaptive Filtering: Algorithms and Practical Implementation, 3rd ed. New York: Springer, 2008.

[2] D. T. M. Slock, "On the convergence behavior of the LMS and the normalized LMS algorithms," IEEE Transactions on Signal Processing, vol. 41, no. 9, pp. 2811-2825, Sep. 1993.

[3] H. C. Shin, A. H. Sayed, and W. J. Song, "Variable step-size NLMS and affine projection algorithms," IEEE Signal Processing Letters, vol. 11, no. 2, pp. 132-135, Feb. 2004.

[4] J. Benesty, H. Rey, L. R. Vega, and S. Tressens, "A nonparametric VSS NLMS algorithm," IEEE Signal Processing Letters, vol. 13, no. 10, pp. 581-584, Oct. 2006.

[5] M. Z. A. Bhotto and A. Antoniou, "A family of shrinkage adaptive filtering algorithms," IEEE Transactions on Signal Processing, vol. 61, no. 7, pp. 1689-1697, Jul. 2013.

[6] K. Ozeki, and T. Umeda, "An adaptive filtering algorithm using an orthogonal projection to an affine subspace and its properties," Electronics Communications Japan, vol. 67-A, no. 5, pp. 19-27, 1984.

[7] M. Chang, N. Kong, and P. Park, "An affine projection algorithm based on reuse time of input vectors," IEEE Signal Processing Letters, vol. 7, no. 8, pp. $750-753$, Aug. 2010.

[8] T. Demeechai, "OBA algorithm with a simplification and new optimal variable convergence factor," Electronics Letters, vol. 31, no. 22, pp. 1907-1908, 1995.

[9] F. Albu, and A. Fagan, "The Gauss-Seidel pseudo affine projection algorithm and its application for echo cancellation", in Proc. of ASILOMAR 2003, Vol. 2, Nov. 2003, pp. 1303 - 1306.

[10] F. Albu, "An Efficient Multichannel Filtered-X Affine Projection Algorithm", IEE Electronics Letters, Vol. 42, Issue 7, pp. 59-60, 2006.

[11] Y. Zakharov and T. Tozer, "Multiplication-free iterative algorithm for LS problem”, Electronics Letters, 40, (9), pp. 567-569, 2004.

[12] M. Bouchard, F. Albu, "The Gauss-Seidel fast affine projection algorithm for multichannel active noise control and sound reproduction systems", International Journal of Adaptive Control and Signal Processing, vol. 19, nr. 2-3, pp. 107-123, 2005.

[13] F. Albu, H.K. Kwan, "Combined echo and noise cancellation based on Gauss-Seidel pseudo affine projection algorithm", Proceedings of IEEE ISCAS 2004, Vol. 3, pp. 505-508, May 2004.

[14] W. Liu and J. C. Principe, "Kernel affine projection algorithms," EURASIP Journal on Advances in Signal Processing, vol. 2008, article ID 784292.

[15] F. Albu, D. Coltuc, K. Nishikawa, and M. Rotaru, "An efficient implementation of the kernel affine projection algorithm", in Proc. of ISPA 2013, September 2013, Trieste, Italy, pp. 342-346.

[16] A. Gonzalez, M. Ferrer, F. Albu and M. de Diego, "Affine projection algorithms: evolution to smart and fast multichannel algorithms and applications", in Proc. of EUSIPCO 2012, August 2012, pp. 1965-1969.

[17] A. Gonzales, F. Albu, M. Ferrer, M. Diego, "Evolutionary and variable step size affine projection algorithms for active noise control ", IET Signal Processing, vol. 7, issue 6, pp. 471-476, 2013.
[18] F. Albu, C. Paleologu and J. Benesty, “A Variable Step Size Evolutionary Affine Projection Algorithm," in Proc. of ICASSP 2011, May 2011, pp. 429-432.

[19] M. Z. A. Bhotto and A. Antoniou, "Affine-Projection-Like AdaptiveFiltering Algorithms Using Gradient-Based Step Size", IEEE Transactions on Circuits and Systems-I: Regular Papers, vol.61, no. 7, pp. 2048-2056, 2014.

[20] D.L. Duttweiler, "Proportionate normalized least-mean-squares adaptation in echo cancellers", IEEE Transactions on Speech and Audio Processing, vol. 8, no. 5, pp. 508-518, 2000.

[21] J. Benesty and S. L. Gay, "An improved pnlms algorithm," in Proc. of ICASSP 2002, vol. 2, 2002, pp. $1881-1884$.

[22] H. Deng and M. Doroslovacki, "Proportionate adaptive algorithms for network echo cancellation," IEEE Transactions on Signal Processing, vol. 54, no. 5, pp. 1794-1803, 2006.

[23] O. Hoshuyama, R.A. Goubran and A. Sugiyama, "A generalized proportionate variable step-size algorithm for fast changing acoustic environments," in Proc. of ICASSP 2004, vol. 4, pp. 161-164.

[24] T. Gansler, J. Benesty, S.L. Gay and M. Sondhi, "A robust proportionate affine projection algorithm for network echo cancellation," in Proc. of ICASSP 2000, pp. 793-796.

[25] F. Albu, C. Paleologu, J. Benesty and S. Ciochina, "A low complexity proportionate affine projection algorithm for echo cancellation," in Proc. of EUSIPCO 2010, August 2010, pp. 6-10.

[26] Z. Yang, Y. R. Zheng and S. L. Grant, "Proportionate affine projection sign algorithms for network echo cancellation," IEEE Transactions on Audio, Speech, and Language Processing, vol. 19, no. 8, pp. 2273-2284, 2011.

[27] F. Albu and H. K. Kwan, "Memory Improved Proportionate Affine Projection Sign Algorithm", IET Electronics Letters, vol. 48, no. 20, pp. 1279-1281, October 2012.

[28] F. Albu and H. Coanda, "A Fast Filtering Proportionate Affine Projection Sign Algorithm”, in Proc. of IEEE SPA 2014, September 2014, pp. 25-30.

[29] T. Waterschoot and M. Moonen, "Fifty years of acoustic echo feedback control: state of the art and future challenges", in Proc. IEEE, vol. 99, no. 2, pp. 288-327, 2011

[30] M. Rotaru, F. Albu and H. Coanda, "A Variable Step Size Modified Decorrelated NLMS Algorithm for Adaptive Feedback Cancellation in Hearing Aids," in Proc. of ISETC 2012, pp. 263-266, 2012.

[31] C. R. C. Nakagawa, S. Nordholm, F. Albu and W.-Y. Yan, "Closed-loop feedback cancellation utilizing two microphones and transform domain processing", in Proc. of ICASSP 2014, pp. 3673-3677.

[32] A. H. Sayed, Fundamentals of Adaptive Filtering. Hoboken, NJ, USA: Wiley, 2003.

[33] F. Strasser, and H. Puder. "Sub-band feedback cancellation with variable step sizes for music signals in hearing aids." in Proc. of ICASSP 2014, pp. 8207-8211, 2014

[34] M. Z. A. Bhotto, M. O. Ahmad, and M. N. S. Swamy, "Robust Shrinkage Affine-Projection Sign Adaptive-Filtering Algorithms for Impulsive Noise Environments, IEEE Transactions on Signal Processing 2014, vol. 62, issue 13, pp. 3349-3359, 2014. 